\title{
Oxaliplatin-incorporated micelles eliminate both cancer stem-like and bulk cell populations in colorectal cancer
}

This article was published in the following Dove Press journal:

International Journal of Nanomedicine

5 December 201 I

Number of times this article has been viewed

\author{
Ke Wang ${ }^{1, *}$ \\ Lina $\operatorname{Liu}^{2, *}$ \\ Tao Zhang' \\ Yong-liang Zhu ${ }^{3}$ \\ Fuming $\mathrm{Qiu}^{4}$ \\ Xian-guo $\mathrm{Wu}^{\prime}$ \\ Xiao-lei Wang' \\ Fu-qiang $\mathrm{Hu}^{5}$ \\ Jian Huang ${ }^{1,4}$ \\ 'Cancer Institute (Key Laboratory of \\ Cancer Prevention and Intervention, \\ National Ministry of Education; \\ Provincial Key Laboratory of \\ Molecular Biology in Medical \\ Sciences), The Second Affiliated \\ Hospital, Zhejiang University \\ School of Medicine; ${ }^{2}$ Department of \\ Pharmacy, Second Affiliated Hospital \\ (Binjiang Branch), Zhejiang University \\ School of Medicine; ${ }^{3}$ Department of \\ Gastroenterology; ${ }^{4}$ Department of \\ Oncology, Second Affiliated Hospital, \\ Zhejiang University School of \\ Medicine; ${ }^{5}$ College of Pharmaceutical \\ Science, Zhejiang University, \\ Hangzhou, China \\ *These authors contributed equally \\ to this work
}

Purpose: The failure of cancer treatments is partly due to the enrichment of cancer stem-like cells (CSLCs) that are resistant to conventional chemotherapy. A novel micelle formulation of oxaliplatin (OXA) encapsulated in chitosan vesicle was developed. The authors postulate that micelle encapsulation of OXA would eliminate both CSLCs and bulk cancer cells in colorectal cancer (CRC).

Experimental design: In this study, using stearic acid-g-chitosan oligosaccharide (CSO-SA) polymeric micelles as a drug-delivery system, OXA-loaded CSO-SA micelles (CSO-SA/OXA) were prepared. Intracellular uptake of CSO-SA/OXA micelles was assessed by confocal microscope. The effects of free OXA, the empty carrier, and CSO-SA/OXA micelles were tested using human CRC cell lines in vitro and in vivo.

Results: The micelles showed excellent internalization ability that increased OXA accumulation both in CRC cells and tissues. Furthermore, CSO-SA/OXA micelles could either increase the cytotoxicity of OXA against the bulk cancer cells or reverse chemoresistance of CSLC subpopulations in vitro. Intravenous administration of CSO-SA/OXA micelles effectively suppressed the tumor growth and reduced $\mathrm{CD} 133^{+} / \mathrm{CD} 24^{+}$cell (putative CRC CSLC markers) compared with free OXA treatment, which caused CSLC enrichment in xenograft tumors $(P<0.05)$.

Conclusion: The results of this study indicate that CSO-SA micelle as a drug-delivery carrier is effective for eradicating CSLCs and may act as a new option for CRC therapy.

Keywords: polymeric micelle, chemotherapy resistance, tumor

\section{Introduction}

Colorectal cancer (CRC) is a prevalent disease worldwide and remains one of the leading causes of mortality. An emerging paradigm has revealed that cancers including CRC may be a stem cell disease. ${ }^{1-4}$ Cancer stem-like cells (CSLCs) are a subpopulation of cells within tumors that have self-renewal and pluripotent capabilities, responsible for tumor initiation, growth, and metastasis. Current approaches for isolation and identification of CSLCs are based on the expression of particular biomarkers or ability of forming spheroids, being indicative of being more tumorigenic and aggressive. ${ }^{5-9}$

Accumulating evidence has shown that the failure of conventional chemotherapy is closely related to the enrichment of CSLCs, which are more resistant than bulk cells to the treatment and drive chemoresistance. ${ }^{10-13}$ Focus has thus been on defining new therapeutic agents for reversal of chemotherapy resistance via targeting CSLCs. Even though great effort has been made in the past few decades, the effect is not desirable. The important fact is that non-CSLCs within a tumor can be transformed 
to CSLCs through epithelial-mesenchymal transition (EMT) and maintain the disease. ${ }^{14,15}$ In addition, it is not yet clear what fraction of tumor cells is CSLCs and therefore needs to be eliminated. These points suggest that a highly desirable strategy is urgently needed that will target both the CSLCs and bulk cancer cells in tumors. ${ }^{16,17}$

Nanotechnology offers one of the innovative approaches to overcome these problems. Polymeric micelles with core-shell structure, formed by self-aggregation of amphiphilic graft or block copolymers, provide a significant advantage for delivering antitumor drugs to solid tumors. ${ }^{18-22}$ Moreover, polymeric micelles are largely accumulated in tumor tissue via the passive "enhanced permeability and retention (EPR) effect."23,24 Among the natural polysaccharides, the stearic acid-g-chitosan oligosaccharide (CSO-SA) micelles have been widely utilized as polymeric drug carriers due to their biocompatibility and biodegradability. ${ }^{25,26} \mathrm{CSO}$ is nontoxic, low immunogenic, and biodegradable, obtained from the enzymatic degradation of chitosan. And SA also has low cytotoxicity as it is mainly composed of fat. CSO-SA micelles are thus used as a carrier for physically or chemically loading antitumor drugs, with excellent tumor cellular uptakes. Furthermore, micelle encapsulation of drug was studied to improve the antitumor activity and reverse multidrug resistance in xenograft tumors. ${ }^{27-30}$ For example, a CSO-SA-based polymeric nanosystem for oxaliplatin (OXA) loading has recently been reported to have obvious antiproliferative effects against breast cancer cells in vitro. ${ }^{31}$

However, the antitumor activity of OXA-loaded micellar nanoparticles in a CRC model has not yet been reported. In particular, OXA is now a standard chemotherapy option for CRC. Clinical studies have demonstrated that combined use of OXA with 5-fluorouracil and leucovorin for metastatic CRC has achieved response rates $>50 \%$ and median survival approaching 2 years, ${ }^{32,33}$ but nearly all responding CRCs eventually developed OXA resistance and progressed within 8 months. ${ }^{34}$ Several hypotheses on OXA-resistant mechanisms currently include decreased drug uptake, impaired DNA adduct formation, induced EMT phenotype, and proven insensitivity to CSLCs. ${ }^{13,35-37}$ Based on the recent assumption that polymeric micelles in drug-delivery systems facilitate antitumor drug uptake and overcome drug resistance, the strong indication of antitumor activity of OXA-loaded CSO-SA (CSO-SA/OXA) micelles in particular needs to be noted and explored, especially in a colorectal CSLC model. In this study, CSO-SA/OXA micelles were prepared and studied in detail to investigate whether they could eliminate CSLCs as well as bulk cancer cells in CRC.

\section{Materials and methods}

\section{Synthesis and characteristics of CSO-SA}

CSO-SA was prepared from a modified procedure developed in the laboratory (College of Pharmaceutical Science, Zhejiang University, China) by Fu-qiang Hu. CSO-SA was synthesized by esterification reaction between amine groups of CSO (molecular weight $18 \mathrm{kDa}$ ) and the carboxyl group of SA $\left(\mathrm{C}_{18} \mathrm{H}_{36} \mathrm{O}_{2}\right.$, Shanghai Chemical Reagent Co, Shanghai, China) in the presence of carbodiimide (EDC). Briefly, SA and $\mathrm{EDC}(\mathrm{SA}: \mathrm{EDC}=3: 20, \mathrm{w} / \mathrm{w})$ were dissolved in ethanol. The solution was stirred and heated to $80^{\circ} \mathrm{C}$ in order to activate the carboxylic acid of SA. After CSO (CSO:SA = 2.4:1.0, $\mathrm{w} / \mathrm{w}$ ) solution was added, the reaction was carried out at $80^{\circ} \mathrm{C}$ under stirring for 4 hours. The reaction solution was dialyzed using a dialysis membrane (molecular weight cutoff [MWCO] $7.0 \mathrm{kDa}$ ) against pure water and then lyophilized. Finally, CSO-SA was obtained, and the degree of amino substitution was tested as described previously. ${ }^{27}$

The critical micelle concentration (CMC) of the synthesized CSO-SA was determined by fluorescence methods. In brief, using pyrene as a probe, fluorescence spectra of CSO-SA micelles were measured by fluorometer (F-2500, Hitachi, Tokyo, Japan), excitation wavelength of which was set at $337 \mathrm{~nm}$. The intensities of the emission were recorded at a range of 360-450 $\mathrm{nm}$. The value of CMC was determined by the emission intensity ratio of the first peak $\left(\mathrm{I}_{1}, 374 \mathrm{~nm}\right)$ to the third peak $\left(\mathrm{I}_{3}, 385 \mathrm{~nm}\right)$.

\section{Preparation of OXA-loaded CSO-SA micelles}

CSO-SA/OXA micelles were prepared through thin film dispersed as previously reported. ${ }^{31}$ A volume of $2.5 \mathrm{~mL}$ of $1 \mathrm{mg} / \mathrm{mL}$ OXA (Sigma-Aldrich, St Louis, MI) solution was added into CSO-SA micelle solution (OXA:CSO-SA = 1:10, $\mathrm{w} / \mathrm{w})$. The mixture was stirred for 30 minutes and added into $2.5 \mathrm{~mL}$ lecithin ethanol solution. The solvent was removed by rotary evaporation at $45^{\circ} \mathrm{C}$. CSO-SA/OXA micelles were prepared by dissolving in $5 \%$ glucose.

\section{Physicochemical properties of OXA-loaded CSO-SA micelles}

The size and its distribution of CSO-SA/OXA micelles were measured by dynamic light scattering (DLS) using a Zetasizer (3000 HS, Malvern Instruments Ltd, Worcestershire, UK). The zeta potential of the micelles was detected by the Zetasizer. 
The morphological examinations of CSO-SA/OXA micelles were determined by transmission electron microscopy (TEM) (JEM-1230, JEOL, Tokyo, Japan). The samples were stained with $2 \%(\mathrm{w} / \mathrm{v})$ phosphotungstic acid and placed on copper grids for viewing by TEM.

The OXA content in the drug-loaded micelles was detected using inductively coupled plasma mass spectrometer (ICP-MS) (Hitachi). In brief, the platinum (Pt) content in CSO-SA/OXA micelle solution and ultrafiltrated solution were measured, respectively. The latter solution was obtained from the ultracentrifugation of CSO-SA/OXA solution, using an ultrafilter centrifuge tube (MWCO $10 \mathrm{kDa}$; Millipore, Bedford, MA) centrifuged at 10,000 rpm for 10 minutes. Pt content in solution was determined by ICP-MS, and then the OXA content was calculated. OXA encapsulation efficiency (EE) was calculated using the following equation: $\mathrm{EE}=$ (mass of OXA encapsulated in micelle/mass of OXA added) $\times 100 \%$.

\section{In-vitro drug release-assay}

In-vitro release profiles of OXA from the CSO-SA/OXA micelles were examined using the dialysis method. CSO-SA/ OXA micelles were mixed with fetal bovine serum (FBS) (CSO-SA/OXA:FBS = 1:9, v:v), resulting in a final OXA concentration of $50 \mu \mathrm{g} / \mathrm{mL}$. The solution was then added into a dialysis bag (MWCO 7.0 kDa), and sealed to a plastic tube containing $10 \mathrm{~mL}$ of FBS. The test was conducted in an incubator shaker at $37^{\circ} \mathrm{C}$, which was shaken horizontally at $60 \mathrm{rpm}$. At various time points, FBS in the tube was collected and replaced with the fresh media. The amount of released drug was determined by ICP-MS. All drug-release tests were performed thrice.

\section{Cell culture and spheroid formation}

CRC cell lines HT29 and SW620 were purchased from the cell bank of the Chinese Academy of Medical Science (China). The HT29 cells were cultured in McCoy's 5A medium (Invitrogen, Carlsbad, CA) and SW620 cells in Leibovitz's L-15 medium (Invitrogen). Media were supplemented with $10 \% \mathrm{FBS}, 100 \mathrm{unit} / \mathrm{mL}$ penicillin and $100 \mu \mathrm{g} / \mathrm{mL}$ streptomycin. Cells were incubated at $37^{\circ} \mathrm{C}$ in a $5 \% \mathrm{CO}_{2}$ humidified incubator.

For the formation of spheroids, cells were cultured in Dulbecco's Modified Eagle Medium: Nutrient Mixture F-12 (Invitrogen) basal serum-free medium supplemented with B27, $20 \mathrm{ng} / \mathrm{mL}$ epidermal growth factor, $10 \mathrm{ng} / \mathrm{mL}$ basic fibroblast growth factor (Invitrogen), $2 \mathrm{mg} / \mathrm{mL}$ bovine serum albumin, $100 \mathrm{unit} / \mathrm{mL}$ penicillin, and $100 \mu \mathrm{g} / \mathrm{mL}$ streptomycin . Single cells prepared from enzymatic dissociation were seeded in 6-well ultra low attachment plates at low densities (500-1000 cells/well) or in 96-well ultra low attachment plates at a density of 100-200 cells/well. After 10-14 days of suspension culture with or without drug, the number of spheroids was counted per 200 cells in primary seeded culture.

\section{Intracellular uptake of OXA-loaded CSO-SA micelles}

The cellular uptake studies were done as previously described. ${ }^{18}$ In brief, CSO-SA/OXA micelles were labeled with fluorescein isothiocyanate (FITC) via the reactive amino group of chitosan and the isothiocyanate group of FITC $(\mathrm{CSO}-\mathrm{SA} / \mathrm{OXA}:$ FITC $=1: 8, \mathrm{~mol} / \mathrm{mol})$. Unreacted FITC was removed using dialysis. HT29 spheroids were seeded at a density of 100 cells/well in a 24-well low adhesion plate (Corning, New York, NY). When grown for 24 hours, cells were then incubated with FITC-labeled CSO-SA/OXA micelle solution in growth medium for an additional 30 minutes, 1 hour, and 2 hours, respectively. After incubation, the cells were washed with phosphatebuffered saline (PBS) thrice, and then the cellular uptake was visualized by confocal microscope (Zeiss LSM 710, Carl Zeiss, Dublin, CA).

\section{Pt accumulation studies of OXA-loaded CSO-SA micelles}

For drug-accumulation studies, dissociated HT29 and SW620 spheroid cells were seeded into $5 \times 10^{4}$ cells/well in a 24-well low adhesion plate. When grown for 24 hours, cells were exposed to OXA and CSO-SA/OXA micelles for various periods ( 2 hours, 4 hours, or 8 hours). After incubation, all cells were washed with ice-cold PBS thrice, harvested, and dissolved in pure water. Total cellular Pt content was determined by ICP-MS, and drug accumulation was then calculated.

The drug-accumulation studies of tumor tissues were investigated in xenograft tumors. When the tumors reached a mean diameter of $10 \mathrm{~mm}$, mice were injected in the tail vein with OXA or CSO-SA/OXA micelle solution. At various time points ( 2 hours, 8 hours, or 24 hours), mice were humanely euthanized and tumors were harvested. The cancer tissues were thoroughly washed four times in PBS solution and crushed in liquid nitrogen. Pt content was detected as above. 


\section{Fluorescence-activated cell analyzing and sorting}

Fluorescent-activated cell analyzing or sorting was performed using standard protocols. Single cells obtained from cell cultures or xenograft tumors were labeled with mouse antihuman CD133/2-PE (Miltenyi-Biotec GmbH, Bergisch Gladbach, Germany) and CD24-FITC (BD Biosciences, San Diego, CA). Mouse immunoglobulin G was used as isotype control. Cells were analyzed by FACSCalibur machine (Becton Dickinson, Franklin Lakes, NJ) or sorted by FACSAriaII (Becton Dickinson).

\section{Viability assays}

The cytotoxicities of CSO-SA micelles, OXA, and CSO-SA/ OXA micelles were assessed by 3-(4,5-dimethylthiazol-2yl)2,5-diphenyltetrazolium bromide (MTS) assay using the CellTiter 96 Aqueous MTS (Promega, Fitchburg, WI). HT29 and SW620 cells were seeded in $100 \mu \mathrm{L}$ of medium at a density of $2 \times 10^{4}$ to $5 \times 10^{4}$ cells/well in 96-well plates. In order to preserve stemness of CSLCs, dissociated spheroids and $\mathrm{CD} 133^{+} / \mathrm{CD} 24^{+}$cells from HT29 and SW620 were cultured in 96-well low adhesion plates (Corning) containing stem-cell medium. After each treatment for 48 hours, the MTS assay was performed according to the manufacturer's instructions.

\section{In-vivo tumorigenicity assay and mouse treatment}

All experiments involving mice were conducted in accordance with the standard protocol approved by the local animal care committee. Dissociated HT29 and SW620 spheroid cells were injected subcutaneously into the flank of 4-5-weekold female nude mice. Two weeks after the injection, mice carrying xenograft tumors were randomly divided into four groups. Each group with six mice was treated intravenously once a week for 4 weeks with CSO-SA/OXA micelles (OXA content $2 \mathrm{mg} / \mathrm{kg}$ ), free OXA ( $2 \mathrm{mg} / \mathrm{kg}$ ), CSO-SA micelles, or $5 \%$ glucose, respectively. Each tumor was measured with a caliper, and the volume calculated using the formula: $\mathrm{V}=1 / 2$ (width ${ }^{2} \times$ length).
At the end of each treatment, tumors were collected from mouse xenografts and washed four times in PBS solution. After being minced into approximately $1 \mathrm{~mm}^{3}$ in size, enzymatic digestion was performed using $1.5 \mathrm{mg} / \mathrm{mL}$ collagenase type IV and $1 \mathrm{mg} / \mathrm{mL}$ hyaluronidase in medium RPMI (Roswell Park Memorial Institute) 1640 (Invitogen) at $37^{\circ} \mathrm{C}$ for about 1 hour. Cells were then obtained through a $40 \mu \mathrm{m}$ mesh and resuspended in PBS, which was used for flow cytometry.

\section{TdT-mediated dUTP nick end labeling (TUNEL) assay}

TUNEL-reaction was performed on 10-mm thick frozen tissue sections using the one-step TUNEL apoptosis assay kit (Beyotime, Shanghai, China) according to the manufacturer's instructions. In brief, samples were fixed with paraformaldehyde for 30 minutes and permeabilized with $0.1 \%$ Triton $^{\circledR}$ X-100 (Dow Chemical Company, Midland, MI) for 10 minutes. The sections were then washed in PBS and incubated with 5-bromo-4-chloro-3-indolyl-phosphate substrate labeled with $\mathrm{Cy}-3$ for 40 minutes at $37^{\circ} \mathrm{C}$. Randomly chosen fields were examined at $\times 400$ magnification using confocal microscope.

\section{Statistical analysis}

Statistical analysis described in the experimental sections was done with GraphPad Prism 5.0 (GraphPad Software Inc, La Jolla, CA) software. Statistical significance was determined by one-way analysis of variance. $P$-values $<0.05$ were regarded to be statistically significant in all cases.

\section{Results \\ Preparation and characteristics of CSO-SA micelles and OXA-loaded CSO-SA micelles}

The degree of amino substitution of the synthesized CSO-SA was measured by 6-trinitrobenzene sulfonic acid test, which was about 5.1\% (Table 1). The aggregation behavior of CSO-SA was confirmed by fluorescence spectroscopy using pyrene as a probe. The value of the CMC was about

Table I Characteristics of CSO-SA and CSO-SA/OXA micelles

\begin{tabular}{lllllll}
\hline Material & $\begin{array}{l}\text { Size by number } \\
(\mathbf{n m})\end{array}$ & PI & $\begin{array}{l}\text { Zeta potential } \\
(\mathbf{m V})\end{array}$ & DAS (\%) & $\begin{array}{l}\text { CMC } \\
(\mathbf{m g} / \mathbf{m L})\end{array}$ \\
\hline CSO-SA & $25.4 \pm 2.3$ & $0.37 \pm 0.01$ & $29.9 \pm 2.9$ & $5.1 \pm 0.3$ & 0.14 & - \\
CSO-SA/OXA & $90.0 \pm 7.0$ & $0.39 \pm 0.03$ & $24.4 \pm 0.4$ & - & - & $60.7 \pm 1.5$ \\
\hline
\end{tabular}

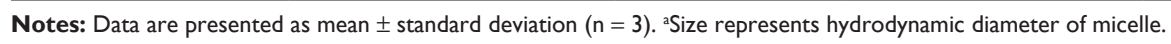

Abbreviations: CMC, critical micelle concentration; CSO-SA, stearic acid-g-chitosan oligosaccharide; EE, encapsulation efficiency; OXA, oxaliplatin; PI, polydispersity index; DAS, degree of amino substitution. 
$0.14 \mathrm{mg} / \mathrm{mL}$, which was consistent with previous results. ${ }^{28,31}$ This low CMC meant that CSO-SA could easily self-assemble to form micelles in aqueous medium and keep the core-shell structure, even under highly diluted conditions.

The mean particle size and zeta potential of CSO-SA micelles and CSO-SA/OXA micelles were measured by the Zetasizer. It was found that the CSO-SA micelles had smaller size $(25.4 \mathrm{~nm})$ and higher positive zeta potential $(29.9 \mathrm{mV})$ in pure water. After the OXA was loaded, the size increased $(90.0 \mathrm{~nm})$ and the zeta potential decreased $(24.4 \mathrm{mV})$. The EE of CSO-SA/OXA micelles reached about $60 \mathrm{wt} \%$ by the present preparation method (Table 1).

The size distribution of micelles was obtained by DLS, and the spherical morphology was detected by TEM imaging (Figure 1A and B). Drug-loaded micelles were obviously larger than that of blank micelles $(P<0.05)$. However, the size of micelles observed in the TEM images was smaller than

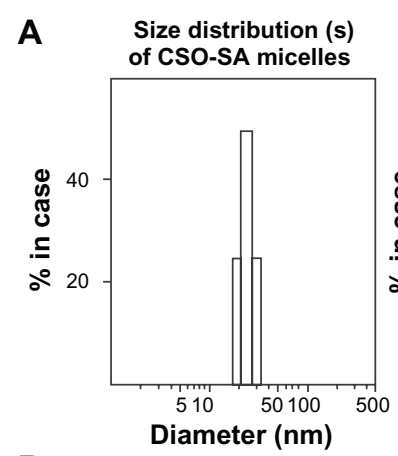

B

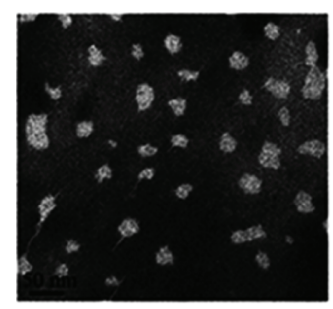

CSO-SA micelles

C

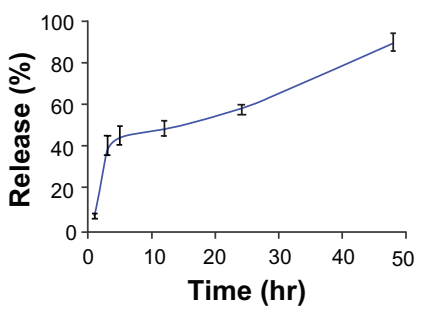

Figure I Characteristics of blank micelles and OXA-loaded CSO-SA micelles. (A) Size distribution of CSO-SA micelles and CSO-SA/OXA micelles. (B) TEM image of CSO-SA micelles and CSO-SA/OXA micelles. TEM image of micelles stained with $4 \%$ uranyl acetate. (Scale bar, $50 \mathrm{~nm}$.) (C) In-vitro drug-release profile of CSO-SA/ OXA micelles in fetal bovine serum at $37^{\circ} \mathrm{C}$.

Note: Data are presented as mean \pm standard deviation.

Abbreviations: CSO-SA, stearic acid-g-chitosan oligosaccharide; OXA, oxaliplatin; TEM, transmission electron microscopy. that obtained by DLS. The reason might be the shrinking of the hydrophilic backbone in the micelles during the drying process of the TEM sample. On the other hand, CSO-SA/ OXA micelles were stable at room temperature or $4^{\circ} \mathrm{C}$, and the micelles had minimal change in the particle size in storage (for 2 weeks at $4^{\circ} \mathrm{C}$ ) as detected by the Zetasizer. The drug-release profile of CSO-SA/OXA micelles was assessed using FBS ( $\mathrm{pH} 7.4$ ) as media. It was shown that about $90 \%$ total OXA was released from micelles at $37^{\circ} \mathrm{C}$ for 48 hours (Figure 1C).

\section{OXA-loaded CSO-SA micelles enhancing $P t$ accumulation in cancer cells and tissues}

The cellular uptake test was carried out in HT29 spheroids using FITC-labeled CSO-SA/OXA micelles. The cells were incubated with FITC-labeled CSO-SA/OXA micelles for 30 minutes, 1 hour, and 2 hours, respectively. The green fluorescence indicated the FITC-labeled CSO-SA/OXA micelles had excellent rapid cellular uptake capability (Figure 2A). The characteristics of micelles were consistent with previous studies. ${ }^{28}$

The reduced drug accumulation was considered as a defense mechanism of tumor cells against Pt drug. To investigate the enhanced cytotoxicity and reversal of drug resistance of CSO-SA/OXA micelles, dissociated spheroids of HT29 and SW620 were exposed to OXA or CSO-SA/OXA micelles at different incubation times, and the Pt content was then determined using ICP-MS. Comparing with parent drug, the accumulation of $\mathrm{Pt}$ concentration increased significantly as OXA was loaded into CSO-SA micelles at each time point (Figure 2B) $(P<0.05)$. It was confirmed that intracellular delivery of OXA increased significantly via the transport mediated by CSO-SA micelles.

The concentration-time profiles of OXA and CSO-SA/ OXA micelles in xenograft tumor tissues were further studied. The intravenous route through the tail vein was selected for the solution injection. It was found that the CSO-SA/OXA had a higher level of Pt accumulation compared with free OXA at each time point in tumor tissues (Figure 2C) $(P<0.05)$.

\section{OXA-loaded CSO-SA micelles effectively suppressing both CSLCs and the bulk cancer cells}

The cytotoxicities of CSO-SA micelles, OXA, and CSO-SA/OXA micelles were evaluated by MTS assay. 

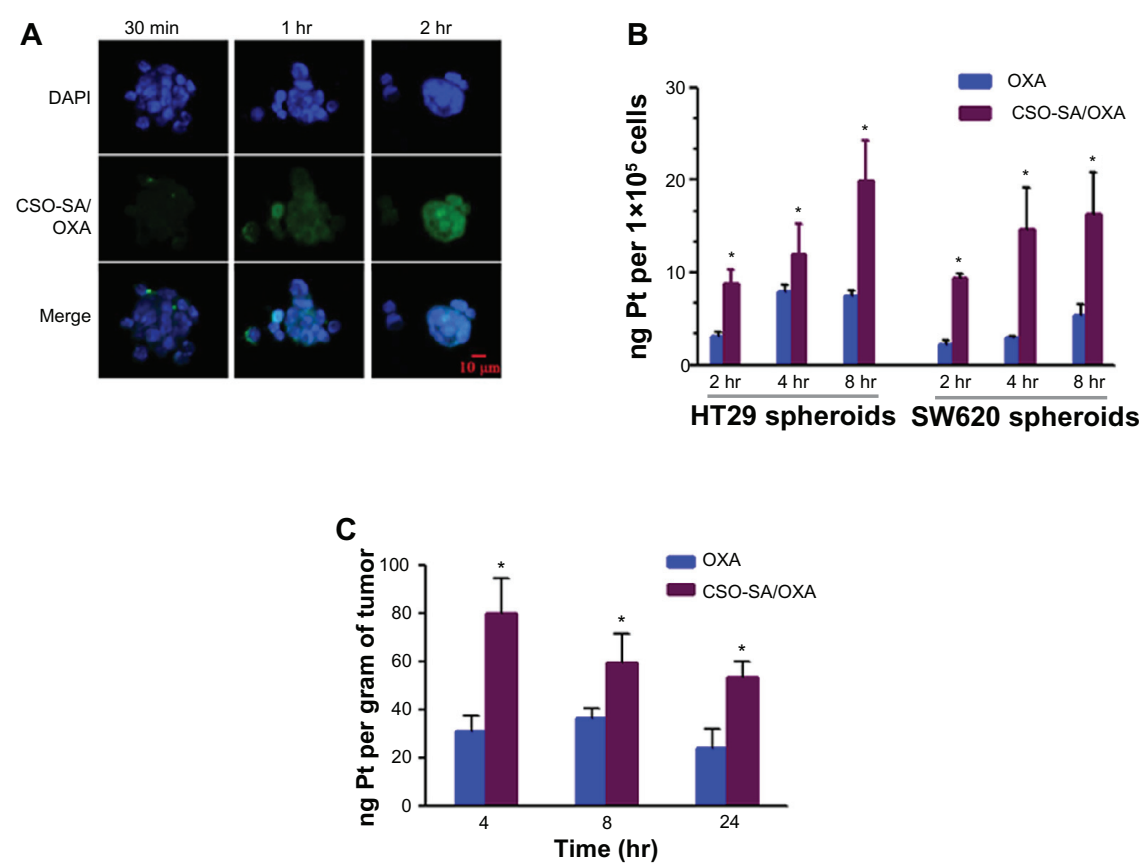

Figure 2 OXA-loaded CSO-SA micelles show excellent internalization ability and enhance Pt accumulation in tumor cells and tissues. (A) Cellular uptake of fluorescein isothiocyanate-labeled CSO-SA/OXA micelles in HT29 spheroids was analyzed by confocal microscope. Images were obtained after cells were incubated with CSO-SA/OXA micelles (OXA content was $10 \mu \mathrm{g} / \mathrm{mL}$ ) for 30 minutes, I hour, and 2 hours, respectively. (B) The intracellular Pt concentrations increased in HT29 and SW620 spheroids treated with CSO-SA/OXA micelles (OXA content was $10 \mu \mathrm{g} / \mathrm{mL})$ compared with free OXA $(10 \mu \mathrm{g} / \mathrm{mL})(*$ denotes $P<0.05)$. Data are presented as mean \pm SD. (C) Intravenous administration of CSO-SA/OXA micelles increased Pt accumulation in xenograft tumors $(*$ denotes $P<0.05)$. Mice received a single injection of free OXA ( $2 \mathrm{mg} / \mathrm{kg}$ ) or CSO-SA/OXA micelles (OXA content was $2 \mathrm{mg} / \mathrm{kg}$ ).

Note: Data are mean \pm SD of three tumors per group.

Abbreviations: CSO-SA, stearic acid-g-chitosan oligosaccharide; DAPI, 4',6-diamidino-2-phenylindole; OXA, oxaliplatin; Pt, platinum; SD, standard deviation.

The $50 \%$ cellular growth inhibition $\left(\mathrm{IC}_{50}\right)$ value of blank CSO-SA micelles in model cells was detected as $320-800 \mu \mathrm{g} / \mathrm{mL}$ (Supplementary table 1). It meant that CSO-SA micelles were relatively low in toxicity and safe as drug carriers. The dose-response curves revealed that CSO-SA/OXA micelles resulted in a three- to fourfold decrease in the $\mathrm{IC}_{50}$ value compared with free OXA in HT29 and SW620 cells (Figure 3A and Supplementary table 1), whereas the $\mathrm{IC}_{50}$ value of CSO-SA/OXA micelles was about tenfold lower than that of free OXA against HT29 and SW620 spheroids, which were obviously OXA resistant (Figure 3B). It was interesting that the $\mathrm{IC}_{50}$ value of CSO-SA/OXA micelles varied from 7.5 to $12.0 \mu \mathrm{g} / \mathrm{mL}$ in all cells, including spheroids (Supplementary table 1). The effects of each treatment on the subpopulations of CRC cells were next examined with putative CSLC markers, including CD133 and CD24. Similar results were observed: CSO-SA/OXA micelles suppressed CD133 ${ }^{+}$ $\mathrm{CD} 24^{+}$cells in CRC cell lines as well (Figure 3C). Taken together, these findings provided the fact that CSO-SA/ OXA micelles reversed free OXA resistance of CSLCs and increased cytotoxicity to both CSLCs and the bulk cancer cells in vitro.

\section{OXA-loaded CSO-SA micelles inhibiting formation of spheroids}

To evaluate whether CSO-SA/OXA micelles could influence formation of spheroids under serum-free conditions, the ability of HT29 and SW620 cells to form spheroids after treatments was examined with different drugs for 10-14 days. As shown in Figure 4A, CSO-SA/OXA micelles inhibited the formation of spheroids under one-third of $\mathrm{IC}_{50}$. It was observed that not only did the number of spheroids significantly decline (Figure 4B) $(P<0.05)$ but the size also reduced. It was worth noting that the concentration of CSO-SA/OXA micelles that were capable of suppressing spheroid formation was threefold lower than that of antiproliferative effects in the MTS assay. These results suggested that CSO-SA/OXA micelles were able to decrease the spheroid formation of cancer cells in vitro.

\section{OXA-loaded CSO-SA micelles enhancing antitumor efficacy and reducing CSLCs enrichment in vivo}

To determine whether CSO-SA/OXA micelles could improve cytotoxicity and influence sensitivity of CSLCs as well as the bulk cancer cells in xenografts, dissociated spheroids of 

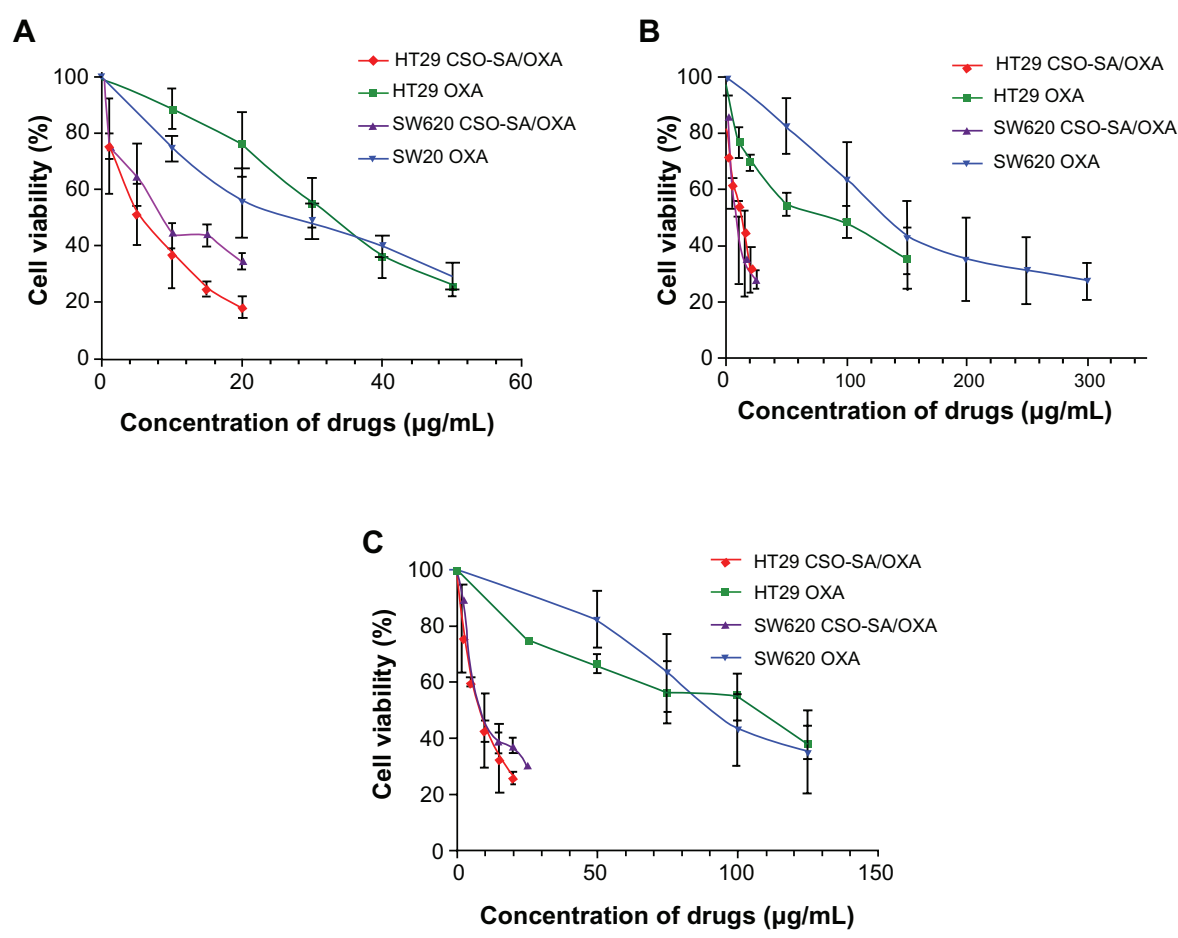

Figure 3 OXA-loaded CSO-SA micelles inhibit proliferation both of colorectal cancer stem-like and bulk cells. The cytotoxicities were analyzed on bulk cells, spheroids, and $\mathrm{CDI} 33^{+} / \mathrm{CD} 24^{+}$sorted cells of $\mathrm{HT} 29$ and SW620 respectively. The cells were treated with different concentrations of OXA and CSO-SA/OXA micelles for 48 hours. The anti-proliferative effect of each drug was measured by MTS assay. (A) Bulk cells of HT29 and SW620. (B) Spheroids. (C) CDI33+/CD24 sorted cells.

Note: Data are presented as mean \pm standard deviation.

Abbreviations: CSO-SA, stearic acid-g-chitosan oligosaccharide; MTS, 3-(4,5-dimethylthiazol-2yl)-2,5-diphenyltetrazolium bromide; OXA, oxaliplatin.

HT29 and SW620 were transplanted into the flank of 4-5week-old female nude mice. Mice were respectively treated with CSO-SA/OXA micelles, OXA, CSO-SA micelles, or $5 \%$ glucose, and tumor growth was measured during the period of 5 weeks. Administration of CSO-SA/OXA micelles marginally inhibited tumor growth, compared with free OXA, CSO-SA micelles, or 5\% glucose (Figure 5A and Supplementary table 2) $(P<0.05)$. In addition, it was found that CSO-SA/OXA micelles did not increase cytotoxicity to mice compared with free OXA as determined by body weight
A
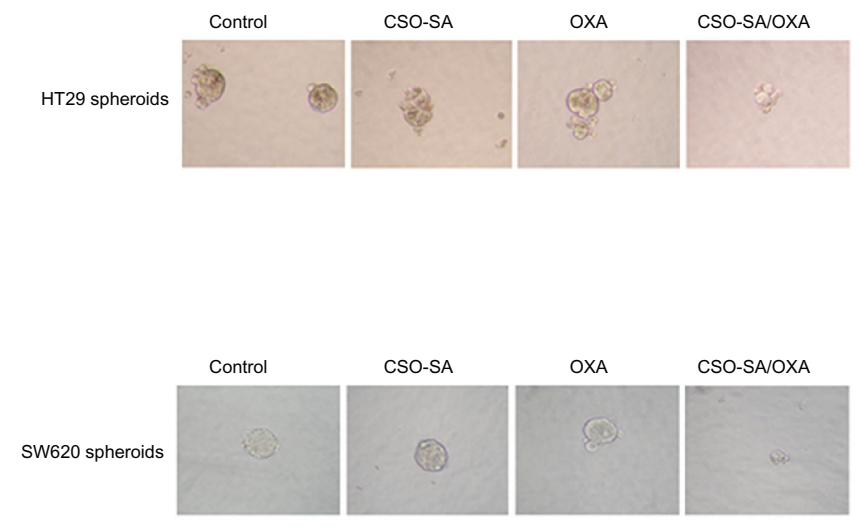
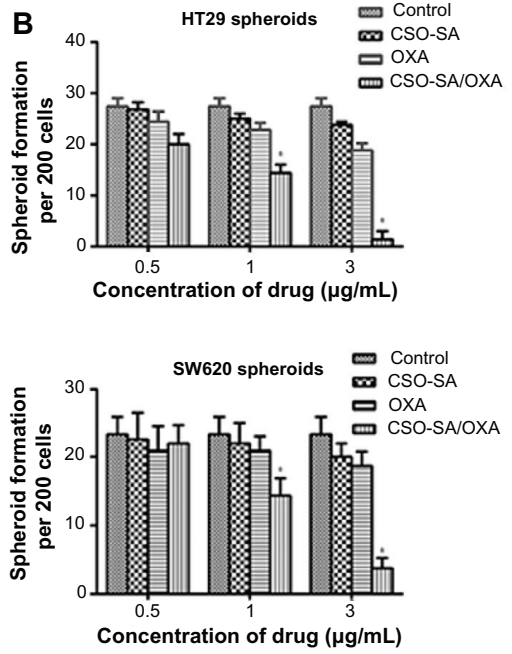

Figure 4 OXA-loaded CSO-SA micelles inhibit formation of spheroids. (A) HT29 and SW620 cells were incubated with OXA (3 $\mu$ g/mL), CSO-SA/OXA micelles (OXA content was $3 \mu \mathrm{g} / \mathrm{mL}$ ), CSO-SA micelles, or $5 \%$ glucose for I0-14 days under spheroid-forming conditions, respectively. The size of the spheroids was then visualized under a microscope. (B) Quantification of spheroid formation with HT29 and SW620 cells treated as in (A).

Note: Data are presented as mean \pm standard deviation (*denotes $P<0.05$ ).

Abbreviations: CSO-SA, stearic acid-g-chitosan oligosaccharide; OXA, oxaliplatin. 
A
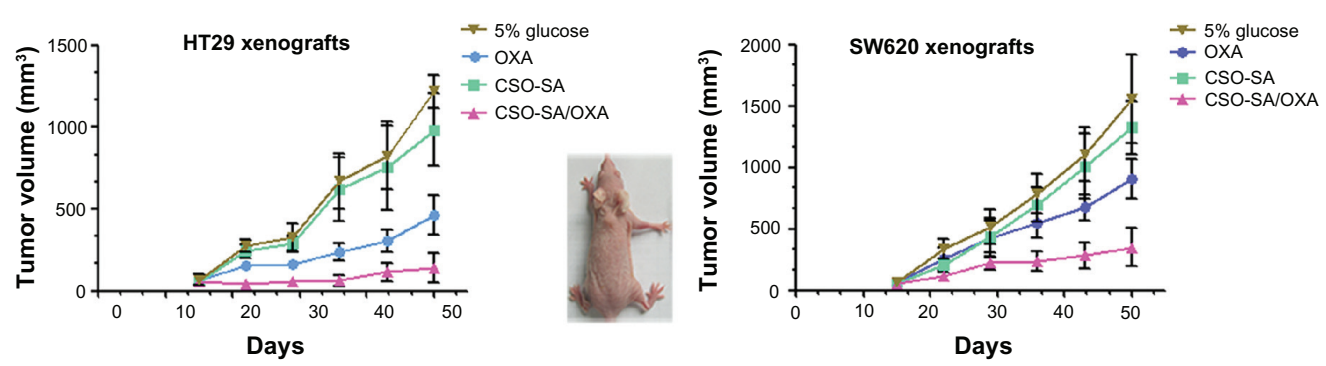

B
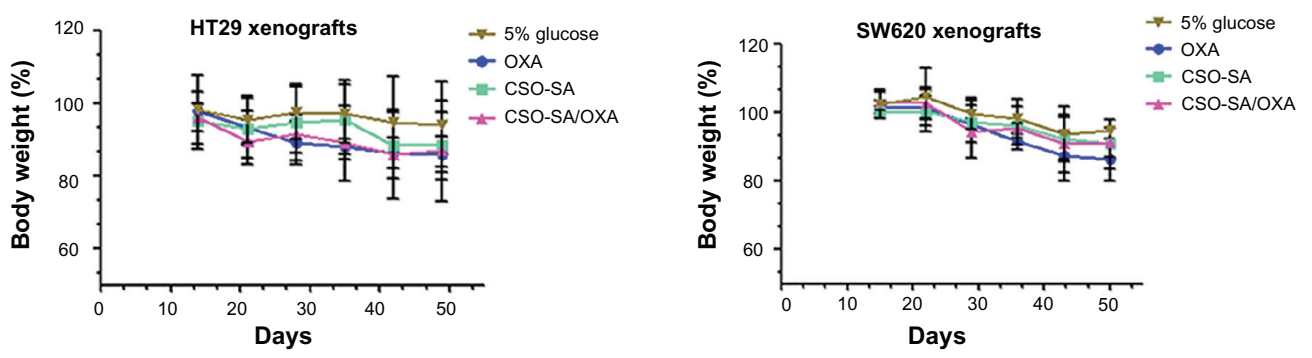

C
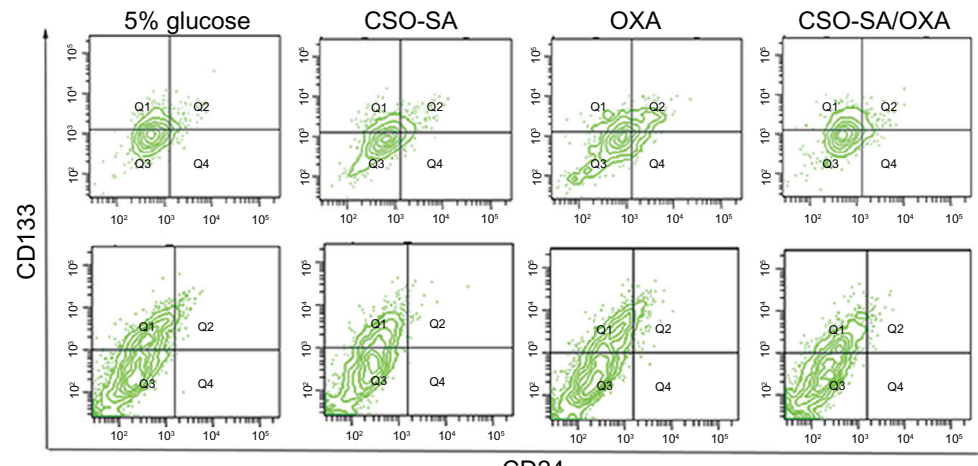

W620 xenografts
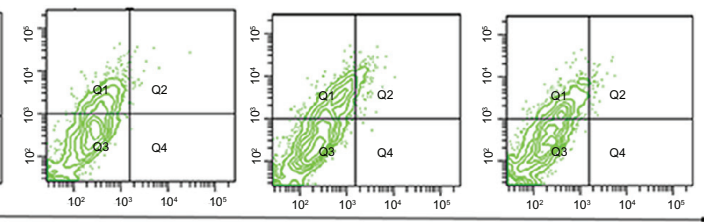

HT29 xenografts

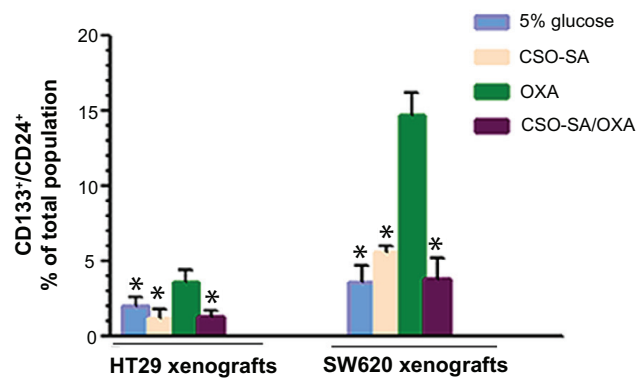

Figure 5 OXA-loaded CSO-SA micelles enhance antitumor efficacy and reduce cancer stem-like cell enrichment in vivo. (A) Reduced size of subcutaneous colon carcinoma tumors derived from spheroid injection of HT29 and SW620 up to 35 days after intravenous treatment with CSO-SA/OXA micelles (OXA content was 2 mg/kg) compared with free OXA ( $2 \mathrm{mg} / \mathrm{kg}$ ), CSO-SA micelles, or $5 \%$ glucose. Data are mean \pm SD of six animals per group. (B) Mice were weighed during the experiment. Each individual mouse's weight was set to $100 \%$ before treatment and bodyweight after treatment was correlated to initial weight. (C) A set of representative flow cytometry plots of the experiments. Q2 covers the region of the double-positive cells. The results show that CSO-SA/OXA micelle treatment did not lead to CDI33 ${ }^{+} / \mathrm{CD} 24^{+}$cell enrichment in tumors.

Note: Data are presented as mean \pm SD (*denotes $P<0.05$, all others versus OXA group).

Abbreviations: CSO-SA, stearic acid-g-chitosan oligosaccharide; OXA, oxaliplatin; QI-Q4, quadrant I-4; SD, standard deviation.

$(P>0.05)$ (Figure 5B). These data indicated that treatment of CSO-SA/OXA micelles could improve therapeutic effects but was well tolerated in vivo.

Moreover, the tumor volume in the free-OXA group was smaller than that of controls, but the former group resulted in the enrichment of $\mathrm{CD} 133^{+} / \mathrm{CD} 24^{+}$stem cell populations
(Figure 5C). In contrast, administration of CSO-SA/OXA micelles significantly reduced CSLC enrichment and increased bulk cancer cell apoptosis (Figure 6A and B). Collectively, these studies demonstrated that CSO-SA/OXA micelles were obviously more effective via directing against both colorectal CSLCs and bulk cancer cells in xenograft tumors. 


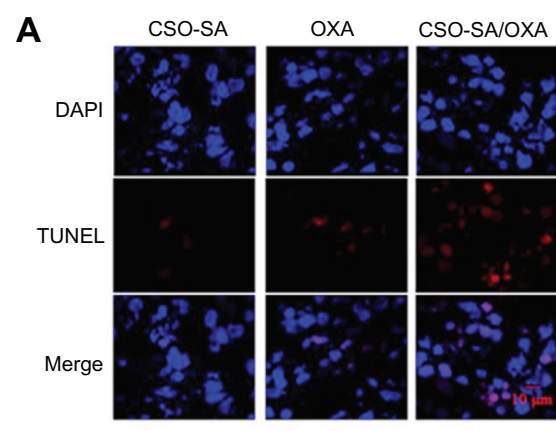

B

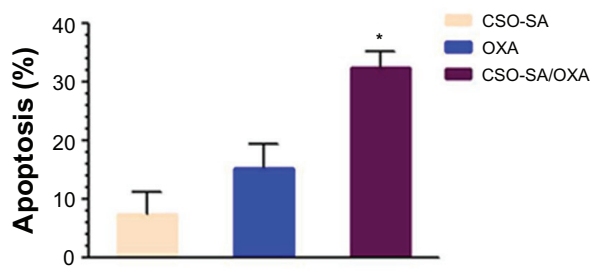

Figure 6 CSO-SA/OXA micelles increase apoptotic events of HT29 xenograft tumors. (A) Enhancement of apoptotic events measured by TUNEL on sections of HT29 xenograft tumors (three per group) treated with CSO-SA/OXA micelles using confocal microscopy analysis. (B) Percentage of apoptotic events measured on sections of (A). A total of $\mathrm{I} \times 10^{5}$ cells per tumor were counted and the percentage of apoptotic cells calculated.

Note: Data are presented as mean \pm standard deviation (*denotes $P<0.05$ ).

Abbreviations: CSO-SA, stearic acid-g-chitosan oligosaccharide; DAPI, 4',6-diamidino-2-phenylindole; OXA, oxaliplatin; TUNEL, TdT-mediated dUTP nick end labeling.

\section{Discussion}

In this study, CSO-SA with 5.1\% degree of amino substitution was synthesized successfully with low CMC. After OXA was loaded, the micellar size was larger in comparison with CSO-SA micelles. Also, the zeta potential of CSO-SA/OXA micelles was lower than that of CSO-SA micelles, which might be caused by increased volume of the micelles. In-vitro drug-release profiles showed that about $90 \%$ total OXA could be released from the micelles in 48 hours. Furthermore, the excellent internalization of the micelles into cancer cells was found in the cellular uptake test, which might result from the positive charge of the micellar shell and the special spatial structure of multiple hydrophobic domains. These findings provided a strong rationale to choose CSO-SA micelle as a drug-delivery carrier.

The objective of the study was to investigate the ability of CSO-SA/OXA micelles to eliminate both CSLCs and the bulk cancer cells in CRC. Although there is no consensus as to the exact criteria that define a CSLC, several approaches are now still to be used to isolate and characterize colorectal CSLCs. Spheroid culture has been used to isolate and expand CSLCs based on the ability of CSLCs to grow in the absence of serum and without attachment to culture plates, whereas differentiated cells fail to survive under the same condition. ${ }^{38-40}$ In the present work, CSO-SA/OXA micelles were found to suppress the spheroid-forming capacity and reverse chemoresistance of spheroids of both HT29 and SW620 cells significantly. Another way is to apply particular cell markers. Even though there are several putative CSLC markers, coexpressions of $\mathrm{CD} 133^{+} / \mathrm{CD} 24^{+}$cells are currently believed to have more characteristics of stemness than single markers. ${ }^{40}$ Previous studies have also proved that as few as $2000 \mathrm{CD}_{133^{+}}$cells are able to form a tumor in NOD/SCID mouse, whereas $10,000 \mathrm{CD} 133^{-}$cells fail ${ }^{41}$ Therefore, in the present study, CD133/CD24 cells were used to evaluate the ability of CSO-SA/OXA micelles inhibiting CSLCs and non-CSLCs. The results revealed that CSO-SA/OXA micelles could increase the cytotoxicity of OXA against the CSLCs and the bulk cancer cells at the similar $\mathrm{IC}_{50}$ values in vitro.

Existing cancer treatments have mostly been developed based on animal models. The injection of human CRC cells into nude mice provides a reliable in-vivo model for studying CRC. ${ }^{3}$ It reveals that CSO-SA/OXA micelles greatly suppress tumor growth by increasing tumor apoptosis and reduced $\mathrm{CD} 133^{+} / \mathrm{CD} 24^{+}$cell populations compared with OXA treatment, which causes CSLC enrichment in xenograft tumors. This notion corresponded with the clinical observations of this study, demonstrating that the proportion of cells expressing CSLCs markers rose greatly after chemotherapy and functioned as the root of tumor recurrence. Even though available agents of selective toxicity for CSLCs were reported, such as salinomycin, ${ }^{16}$ and sulforaphane, ${ }^{42}$ it was still hard to achieve remarkable effect in eradicating tumors. The exact mechanism needs to be further explored.

To the best knowledge of the authors of this paper, this is the first study to show that CSO-SA/OXA micelles can directly eliminate both CSLCs and non-CSLCs in tumors, which provides advantages and clues to address the concern. The authors believe that there are two possible reasons that may explain the results. One is that the effective accumulation and sustained release of the OXA from CSO-SA/OXA into the tumor tissues lead to the suppression of the tumor growth and the increase of apoptosis in tumor cells. Another explanation is that CSO-SA/OXA micelles may overcome adenosine triphosphate-binding cassette transporters in CSLCs and deliver the OXA to cell nucleus, which intercalates DNA and facilitates the antitumor efficacy. 


\section{Acknowledgments}

The authors are grateful for financial support from the joint project of Zhejiang Province and Ministry of Health (Grant No WKJ20092024 to HJ), Zhejiang Provincial Natural Science Foundation of China (Grant No. Z2100366 to HJ and J20091440 to XG Wu), The 151 Talent Project of Zhejiang Province (HJ), and Zhejiang Provincial Program for the Cultivation of High-level Innovative Health Talents (HJ).

\section{Disclosure}

The authors declare no conflicts of interest in relation to this paper.

\section{References}

1. Dean M, Fojo T, Bates S. Tumour stem cells and drug resistance. Nat Rev Cancer. 2005;5(4):275-284.

2. Reya T, Morrison SJ, Clarke MF, Weissman IL. Stem cells, cancer, and cancer stem cells. Nature. 2001;414(6859):105-111.

3. Todaro M, Alea MP, Di Stefano AB, et al. Colon cancer stem cells dictate tumor growth and resist cell death by production of interleukin- 4 . Cell Stem Cell. 2007;1(4):389-402.

4. Dalerba P, Cho RW, Clarke MF. Cancer stem cells: models and concepts. Annu Rev Med. 2007;58:267-284.

5. Dalerba P, Dylla SJ, Park IK, et al. Phenotypic characterization of human colorectal cancer stem cells. Proc Natl Acad Sci U S A. 2007; 104(24):10158-10163.

6. Yeung TM, Gandhi SC, Wilding JL, Muschel R, Bodmer WF. Cancer stem cells from colorectal cancer-derived cell lines. Proc Natl Acad Sci U S A. 2010;107(8):3722-3727.

7. Ding XW, Wu JH, Jiang CP. ABCG2: a potential marker of stem cells and novel target in stem cell and cancer therapy. Life Sci. 2010;86(17-18):631-637.

8. Pang R, Law WL, Chu AC, et al. A subpopulation of CD26+ cancer stem cells with metastatic capacity in human colorectal cancer. Cell Stem Cell. 2010;6(6):603-615.

9. Charafe-Jauffret E, Ginestier C, Iovino F, et al. Aldehyde dehydrogenase 1-positive cancer stem cells mediate metastasis and poor clinical outcome in inflammatory breast cancer. Clin Cancer Res. 2010;16(1): 45-55.

10. Gupta PB, Chaffer CL, Weinberg RA. Cancer stem cells: mirage or reality? Nat Med. 2009;15(9):1010-1012.

11. Fan X, Ouyang N, Teng H, Yao H. Isolation and characterization of spheroid cells from the HT29 colon cancer cell line. Int $J$ Colorectal Dis. 2011;26(10):1279-1285.

12. Lim MC, Song YJ, Seo SS, Yoo CW, Kang S, Park SY. Residual cancer stem cells after interval cytoreductive surgery following neoadjuvant chemotherapy could result in poor treatment outcomes for ovarian cancer. Onkologie. 2010;33(6):324-330.

13. Rich JN, Bao S. Chemotherapy and cancer stem cells. Cell Stem Cell. 2007;1(4):353-355.

14. Mani SA, Guo W, Liao MJ, et al. The epithelial-mesenchymal transition generates cells with properties of stem cells. Cell. 2008;133(4): 704-715.

15. Yang AD, Fan F, Camp ER, et al. Chronic oxaliplatin resistance induces epithelial-to-mesenchymal transition in colorectal cancer cell lines. Clin Cancer Res. 2006;12(14):4147-4153.

16. Gupta PB, Onder TT, Jiang G, et al. Identification of selective inhibitors of cancer stem cells by high-throughput screening. Cell. 2009; 138(4):645-659.

17. Schultz PG, Dubrovska A, Elliott J, et al. Combination therapy targeting both tumor-initiating and differentiated cell populations in prostate carcinoma. Clin Cancer Res. 2010;16(23):5692-5702.
18. Du YZ, Weng Q, Yuan H, Hu FQ. Synthesis and antitumor activity of stearate-g-dextran micelles for intracellular doxorubicin delivery. ACS Nano. 2010;4(11):6894-6902.

19. Du YZ, Cai LL, Li J, et al. Receptor-mediated gene delivery by folic acid-modified stearic acid-grafted chitosan micelles. Int $J$ Nanomedicine. 2011;6:1559-1568.

20. Jeong YI, Kim do H, Chung CW, et al. Doxorubicin-incorporated polymeric micelles composed of dextran-b-poly(DL-lactide-coglycolide) copolymer. Int J Nanomedicine. 2011;6:1415-1427.

21. Wu Y, Wang W, Chen Y, et al. The investigation of polymer-siRNA nanoparticle for gene therapy of gastric cancer in vitro. Int $J$ Nanomedicine. 2010;5:129-136.

22. Diao YY, Li HY, Fu YH, et al. Doxorubicin-loaded PEG-PCL copolymer micelles enhance cytotoxicity and intracellular accumulation of doxorubicin in adriamycin-resistant tumor cells. Int J Nanomedicine. 2011;6:1955-1962.

23. Talelli M, Iman M, Rijcken CJ, van Nostrum CF, Hennink WE. Targeted core-crosslinked polymeric micelles with controlled release of covalently entrapped doxorubicin. J Control Release. 2010;148(1):121-122.

24. Matsumura Y, Maeda H. A new concept for macromolecular therapeutics in cancer chemotherapy: mechanism of tumoritropic accumulation of proteins and the antitumor agent smancs. Cancer Res. 1986;46(12):6387-6392.

25. Hu FQ, Ren GF, Yuan H, Du YZ, Zeng S. Shell cross-linked stearic acid grafted chitosan oligosaccharide self-aggregated micelles for controlled release of paclitaxel. Colloids Surf B Biointerfaces. 2006; 50(2):97-103

26. Hyung Park J, Kwon S, Lee M, et al. Self-assembled nanoparticles based on glycol chitosan bearing hydrophobic moieties as carriers for doxorubicin: in vivo biodistribution and anti-tumor activity. Biomaterials. 2006;27(1):119-126.

27. Hu FQ, Wu XL, Du YZ, You J, Yuan H. Cellular uptake and cytotoxicity of shell crosslinked stearic acid-grafted chitosan oligosaccharide micelles encapsulating doxorubicin. Eur J Pharm Biopharm. 2008; 69(1):117-125.

28. Hu FQ, Liu LN, Du YZ, Yuan H. Synthesis and antitumor activity of doxorubicin conjugated stearic acid-g-chitosan oligosaccharide polymeric micelles. Biomaterials. 2009;30(36):6955-6963.

29. Du YZ, Wang L, Yuan H, Hu FQ. Linoleic acid-grafted chitosan oligosaccharide micelles for intracellular drug delivery and reverse drug resistance of tumor cells. Int J Biol Macromol. 2011;48(1): 215-222.

30. Zhang XG, Miao J, Dai YQ, Du YZ, Yuan H, Hu FQ. Reversal activity of nanostructured lipid carriers loading cytotoxic drug in multi-drug resistant cancer cells. Int J Pharm. 2008;361(1-2):239-244.

31. Xu YY, Du YZ, Yuan H, Liu LN, Niu YP, Hu FQ. Improved cytotoxicity and multidrug resistance reversal of chitosan based polymeric micelles encapsulating oxaliplatin. J Drug Target. 2011;19(5): 344-353.

32. Alberts SR, Horvath WL, Sternfeld WC, et al. Oxaliplatin, fluorouracil, and leucovorin for patients with unresectable liver-only metastases from colorectal cancer: a North Central Cancer Treatment Group Phase II study. J Clin Oncol. 2005;23(36):9243-9249.

33. Cassidy J, Tabernero J, Twelves C, et al. XELOX (capecitabine plus oxaliplatin): active first-line therapy for patients with metastatic colorectal cancer. J Clin Oncol. 2004;22(11):2084-2091.

34. Goldberg RM, Sargent DJ, Morton RF, et al. A randomized controlled trial of fluorouracil plus leucovorin, irinotecan, and oxaliplatin combinations in patients with previously untreated metastatic colorectal cancer. J Clin Oncol. 2004;22(1):23-30.

35. Zhou BB, Zhang H, Damelin M, Geles KG, Grindley JC, Dirks PB. Tumour-initiating cells: challenges and opportunities for anticancer drug discovery. Nat Rev Drug Discov. 2009;8(10):806-823.

36. Trumpp A, Wiestler OD. Mechanisms of Disease: cancer stem cells targeting the evil twin. Nat Clin Pract Oncol. 2008;5(6):337-347.

37. Johannessen TC, Bjerkvig R, Tysnes BB. DNA repair and cancer stem-like cells - potential partners in glioma drug resistance? Cancer Treat Rev. 2008;34(6):558-567. 
38. Cammareri P, Lombardo Y, Francipane MG, Bonventre S, Todaro M, Stassi G. Isolation and culture of colon cancer stem cells. Methods Cell Biol. 2008;86:311-324.

39. Ricci-Vitiani L, Lombardi DG, Pilozzi E, et al. Identification and expansion of human colon-cancer-initiating cells. Nature. 2007; 445(7123):111-115.

40. Vermeulen L, Todaro M, de Sousa Mello F, et al. Single-cell cloning of colon cancer stem cells reveals a multi-lineage differentiation capacity. Proc Natl Acad Sci U S A. 2008;105(36):13427-13432.
41. Wang YK, Zhu YL, Qiu FM, et al. Activation of Akt and MAPK pathways enhances the tumorigenicity of CD133+ primary colon cancer cells. Carcinogenesis. 2010;31(8):1376-1380.

42. Li Y, Zhang T, Korkaya H, et al. Sulforaphane, a dietary component of broccoli/broccoli sprouts, inhibits breast cancer stem cells. Clin Cancer Res. 2010;16(9):2580-2590. 


\section{Supplementary tables}

Table SI IC I0 $_{50}$ values of CSO-SA micelles, OXA, and CSO-SA/ OXA micelles against various cells $(\mu \mathrm{g} / \mathrm{mL})$

\begin{tabular}{lccc}
\hline Cells & \multicolumn{1}{c}{ OXA } & CSO-SA/OXA & \multicolumn{1}{c}{ CSO-SA } \\
\hline HT29 cells & $33.1 \pm 1.2$ & $7.6 \pm 0.7$ & $328.2 \pm 46.8$ \\
SW620 cells & $30.3 \pm 1.8$ & $11.5 \pm 1.2$ & $429.5 \pm 36.3$ \\
HT29 spheroids & $93.3 \pm 8.3$ & $11.9 \pm 0.7$ & $773.0 \pm 95.3$ \\
SW620 spheroids & $101.1 \pm 7.1$ & $10.5 \pm 1.3$ & $736.1 \pm 55.8$ \\
HT29 cells & $98.7 \pm 6.3$ & $9.8 \pm 1.1$ & $416.0 \pm 8.6$ \\
(CDI33+/CD24 $)$ & & & \\
SW620 cells & $99.7 \pm 7.2$ & $11.1 \pm 1.1$ & $375.5 \pm 6.6$ \\
(CDI33 $/$ CD24 $\left.24^{+}\right)$ & & &
\end{tabular}

Notes: The concentration of CSO-SA/OXA micelles was OXA-equivalent concentration. Data are presented as mean \pm standard deviation $(n=3)$.

Abbreviations: $\mathrm{IC}_{50}$, half-maximal inhibitory concentration; CSO-SA, stearic acidg-chitosan oligosaccharide; OXA, oxaliplatin.
Table S2 Relative tumor inhibitory rate

\begin{tabular}{|c|c|c|}
\hline \multirow[t]{2}{*}{ Material } & \multicolumn{2}{|l|}{ T/C (\%) } \\
\hline & $\begin{array}{l}\text { HT29 xenograft } \\
\text { tumors }\end{array}$ & $\begin{array}{l}\text { SW620 xenograft } \\
\text { tumors }\end{array}$ \\
\hline CSO-SA & $93.4 \pm 38.2$ & $89.8 \pm 10.5$ \\
\hline OXA & $42.1 \pm 18.4$ & $55.6 \pm 15.6$ \\
\hline CSO-SA/OXA & $13.8 \pm 10.1$ & $23.2 \pm 11.0$ \\
\hline
\end{tabular}

Notes: Relative tumor inhibitory rate (T/C) was calculated as follows: $100 \% \times$ (experimental relative tumor volume/control relative tumor volume). Data are presented as mean \pm standard deviation $(n=6)$.

Abbreviations: CSO-SA, stearic acid-g-chitosan oligosaccharide; OXA, oxaliplatin.

\section{Publish your work in this journal}

The International Journal of Nanomedicine is an international, peerreviewed journal focusing on the application of nanotechnology in diagnostics, therapeutics, and drug delivery systems throughout the biomedical field. This journal is indexed on PubMed Central, MedLine, CAS, SciSearch $\AA$, Current Contents ${ }^{\circledR} /$ Clinical Medicine,
Journal Citation Reports/Science Edition, EMBase, Scopus and the Elsevier Bibliographic databases. The manuscript management system is completely online and includes a very quick and fair peer-review system, which is all easy to use. Visit http://www.dovepress.com/ testimonials.php to read real quotes from published authors. 